\title{
New approaches to the synthesis of pyridinium $N$-heteroarylaminides
}

\author{
Marta Córdoba, M. Luisa Izquierdo, Julio Alvarez-Builla* \\ Departamento de Química Orgánica, Universidad de Alcalá, Alcalá de Henares, 28871 Madrid, Spain
}

\section{A R T T I C L E I N F O}

\section{Article history:}

Received 13 May 2008

Accepted 6 June 2008

Available online 10 June 2008

\begin{abstract}
A B S T R A C T
Different substituted pyridinium $\mathrm{N}$-heteroarylaminides have been prepared in one step from $\mathrm{N}$-aminopyridinium iodide and the corresponding heteroaryl halide by two alternative routes. The use of Pd catalysis allowed the easy preparation of products from the less reactive haloheterocycles. The use of water as a solvent in conjunction with microwave heating dramatically diminishes the reaction time without having an adverse effect on reaction yields.
\end{abstract}

(c) 2008 Elsevier Ltd. All rights reserved.

\section{Introduction}

Mesomeric betaines are neutral conjugated molecules that can only be represented by canonical formulae in which two opposite charges are delocalized within the $\pi$-electron system. ${ }^{1}$ Some heterocyclic betaines are useful in medicinal chemistry because of their pharmacological activities and low toxicity. ${ }^{2}$ Moreover, the structural features of this class of compounds suggest a high probability of interactions with biomolecules-e.g., DNA, proteins-and they can therefore cross biological membranes in vivo. ${ }^{3}$

Conjugated heterocyclic $N$-ylides 1 (Fig. 1 ), a subgroup of mesomeric betaines, have been widely used as building blocks for the synthesis of fused heterocyclic systems and natural products. This application is due mostly to their 1,3 -dipolar character, ${ }^{4}$ which allows cycloaddition processes to take place efficiently. Today, cycloimmonium ylides-including azomethine imines, usually described as $\mathrm{N}$-aminides 1c (Fig. 1 ) -are involved in a wide range

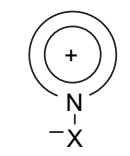

1a $\left(\mathrm{X}: \mathrm{CR}_{2}\right) N$-ylides 1b $(X: O) \quad N$-oxides 1c (X: NR) $N$-aminides

Figure 1.

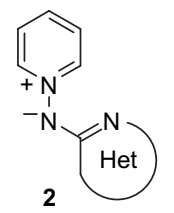

PYRIDINIUM HETEROARYL STABILIZED AMINIDES

\footnotetext{
* Corresponding author. Tel.: +34 9188546 06; fax: +34 918854686 .

E-mail address: julio.alvarez@uah.es (J. Alvarez-Builla).
}

of synthetically useful reactions in the field of heterocyclic chemistry. ${ }^{2,5}$

For several years, part of our research program has been devoted to the study of pyridinium heteroaryl-stabilized aminides 2 (Fig. 1). These compounds have proven to be useful intermediates in heterocyclic synthesis and this is due to their particular structure, in which a positively charged pyridinium ring is linked to a negatively charged 2-aminoheteroaryl moiety. ${ }^{6}$ The whole structure is stable and, having compensated charges, is lipophilic and soluble in moderately polar solvents.

The synthesis of $\mathrm{N}$-aminide intermediates 2 has commonly been performed by the reaction of 2,4-dinitrophenylpyridinium chloride with the corresponding 2-heteroaryl hydrazine in a typical ANRORC process that yields the hydrazone. Acid catalysis then produces the pyridinium salts, which are finally transformed into the $\mathrm{N}$-aminides 2 by treatment with base. This method, ${ }^{6 \mathrm{~b}}$ adapted from that previously described by Beyer, ${ }^{7}$ is suitable for commercially available heteroaryl-derivatives-usually the simpler pyridylhydrazines.

Recently, we developed a method to prepare pyridinium $\mathrm{N}$-heteroarylaminides in one step from the commercially available $\mathrm{N}$-aminopyridinium iodide $\mathbf{3}$ and the corresponding heteroaryl chloride $\mathbf{4}(\mathrm{X}=\mathrm{Cl})$ (Scheme 1$){ }^{6 \mathrm{j}}$ The procedure proved to be useful on using $\pi$-deficient heteroaryl chlorides that are more activated than 2-chloropyridine, but the procedure did not work when the heterocycle was less activated for nucleophilic substitution.

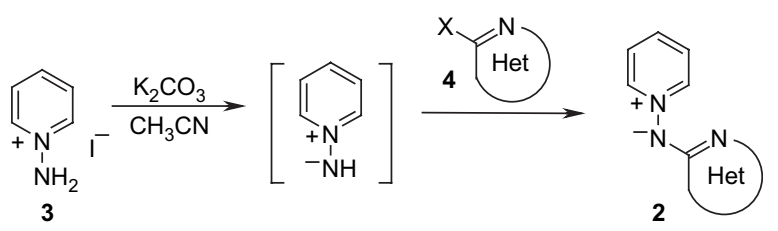

Scheme 1. 
Table 1

Comparative yields for compounds $\mathbf{2 a}-\mathbf{a d}$

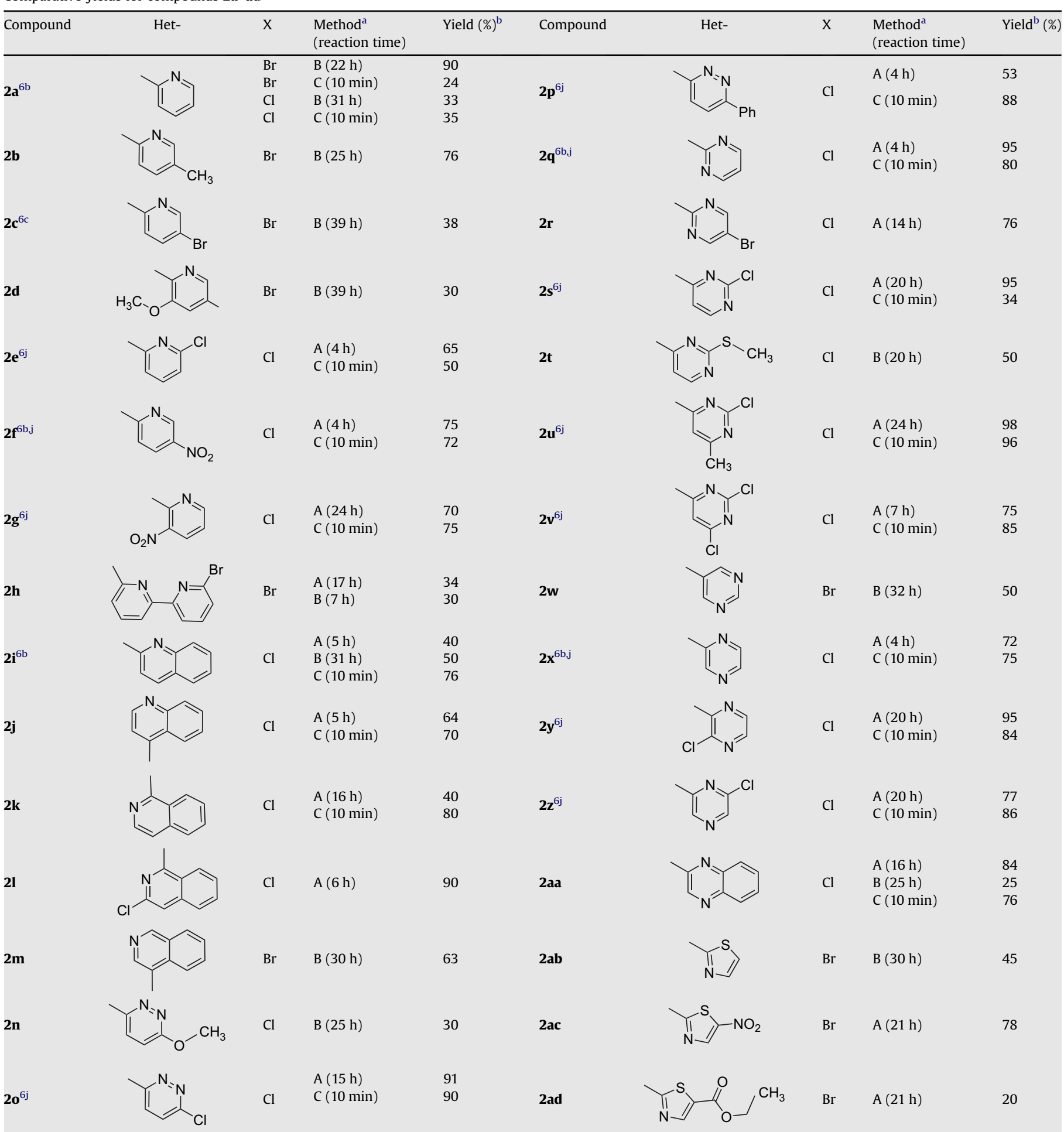

${ }^{\text {a }}$ Method A: $N$-aminopyridinium iodide ( $\left.4.5 \mathrm{mmol}\right)$, aryl halide $(4.7 \mathrm{mmol})$, and potassium carbonate (13.5 mmol) in acetonitrile as solvent. Method B: $N$-aminopyridinium

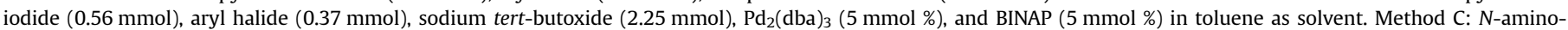
pyridinium iodide $(0.37 \mathrm{mmol})$, aryl halide $(0.39 \mathrm{mmol})$, and potassium carbonate $(1.12 \mathrm{mmol})$ in water at $170{ }^{\circ} \mathrm{C}$ using $\mathrm{MW}$.

b Yields for isolated pure product.

\section{Results and discussion}

In attempts to apply the previously reported one-step synthesis of pyridinium $N$-heteroarylaminides 2 to other types of stabilizing heterocycles, we found that bicyclic haloazines like 2- and 4chloroquinoline, 1-chloroisoquinoline, 1,3-dichloroisoquinoline, and 2-chloroquinoxaline reacted with $\mathrm{N}$-aminopyridinium iodide to yield the corresponding $N$-aminides $\mathbf{2 i}-\mathbf{l}$ and 2aa (see Table 1 and Scheme 2, method A). The result was consistent with the fact that halo-benzoheterocycles are more susceptible to nucleophilic substitution processes. The best yields were obtained for compounds $\mathbf{2 1}$ and 2aa, when a more reactive heteroaryl chloride was 
employed. Two new heteroarylaminides, 2ac and 2ad, stabilized by a thiazole ring were also obtained by this method starting from the corresponding bromo-derivatives $4(\mathrm{X}=\mathrm{Br})$. As expected, the electronic effect of substituents was clear, and 2-bromo-5-nitrothiazole gave a better yield (78\%) than ethyl 2-bromothiazole-5-carboxylate (see Table 1). Aminide $\mathbf{2 h}$ was obtained in an attempt to achieve double coupling on 6,6'-dibromo[2,2']bipyridinyl. Compound $\mathbf{2 r}$, previously prepared by bromination of $\mathbf{2 q},{ }^{6 \mathrm{c}, \mathrm{r}}$ was also obtained in one step and with an increased yield from 5-bromo-2chloropyrimidine.

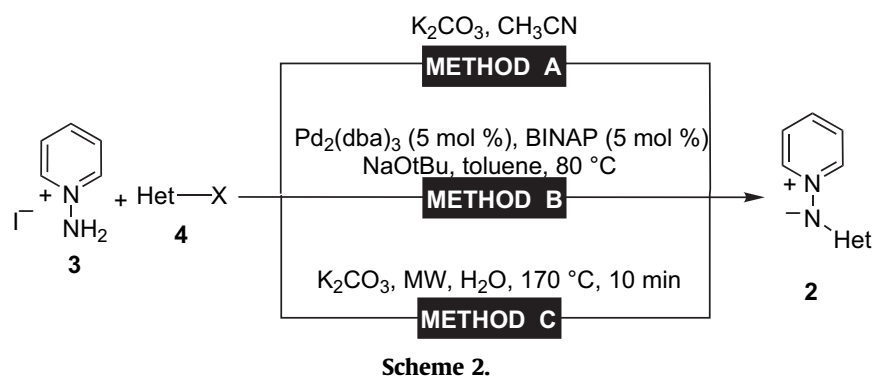

With the aim of expanding the synthesis of pyridinium $\mathrm{N}$-heteroarylaminides $\mathbf{2}$, and bearing in mind that palladium-catalyzed amination of aryl halides has become a versatile method for the preparation of arylamines, ${ }^{8}$ we accomplished the formation of a $\mathrm{C}-\mathrm{N}$ bond between several haloheterocycles and $\mathrm{N}$-aminopyridinium iodide using a palladium-catalyzed amination process (Scheme 2, method B).

2-Bromopyridine, a substrate that did not yield aminide 2a by the conventional method, ${ }^{6 j}$ was chosen for the initial amination experiments in toluene at $80-110^{\circ} \mathrm{C} .^{9}$ The use of the soft bases $\mathrm{Cs}_{2} \mathrm{CO}_{3}$ or $\mathrm{K}_{2} \mathrm{CO}_{3},{ }^{10}$ which produce good results in Suzuki coupling on pyridinium $N$-haloheteroarylaminides, ${ }^{6 \mathrm{k}, \mathrm{n}, \mathrm{q}}$ in conjunction with $\mathrm{Pd}_{2}(\mathrm{dba})_{3}, \mathrm{Pd}(\mathrm{OAc})_{2}$ or $\mathrm{Pd}\left(\mathrm{PPh}_{3}\right)_{4}$ as catalysts and in the presence or absence of complexing agents, e.g., 2,2'-bis(diphenylphosphino)1,1'-binaphthyl (BINAP) or 2-(di-tert-butylphosphine)biphenyl, did not produce the desired coupling. The first encouraging result was achieved with $\mathrm{N}$-aminopyridinium iodide (1.5 equiv) and 2-bromopyridine ( 1 equiv) in toluene at $80{ }^{\circ} \mathrm{C}$, with $\mathrm{Pd}_{2}(\mathrm{dba})_{3}(5 \mathrm{~mol} \%)$ as the catalyst and 2-(di-tert-butylphosphine)biphenyl (5 mol\%) with sodium tert-butoxide $\left(\mathrm{NaO}^{t} \mathrm{Bu}\right)$ (6 equiv) as the base. ${ }^{11}$ Under these conditions, compound $\mathbf{2 a}^{6 \mathrm{~b}}$ was obtained in $33 \%$ yield. A change in the phosphine to BINAP improved the yield of 2 a to $90 \%$. On applying these standard conditions (method B) to other commercially available bromo- or chloro-heterocycles, compounds $\mathbf{2 h}-$ i, 2aa and some new stabilized pyridinium $N$-heteroarylaminides (2b-d, 2n, 2t, and 2ab) were obtained in moderate to low yields. In general, chloro-derivatives gave rise to lower yields (see Table 1). Two non-stabilized pyridium aminides (2w and $\mathbf{2 m}$ ) were also prepared in this way.

Finally, a change in the synthetic route for aminides $\mathbf{2}$ to a more environmentally friendly methodology was investigated. The use of water in conjunction with microwaves in organic synthesis has become increasingly popular in recent years ${ }^{12}$ and we therefore decided to test the reaction between $\mathrm{N}$-aminopyridinium iodide, anhydrous potassium carbonate, and chloro-heterocycles in water in a microwave system.

The study began with 2-chloroquinoline, a substrate that produced $2 \mathbf{i}$ in reasonable yields by methods A and B (see Table 1 and Scheme 2) and was selected here to optimize reaction time and temperature. Preliminary results showed that, at $100{ }^{\circ} \mathrm{C}$, changes in reaction time from 10 to $20 \mathrm{~min}$ did not improve reaction yields, as determined by HPLC-MS. However, when the temperature was raised to $150{ }^{\circ} \mathrm{C}$ the conversion increased from 17 to $45 \%$ in $10 \mathrm{~min}$.
On the basis of these results, the reaction time was fixed at $10 \mathrm{~min}$ and additional optimization experiments were performed to modify the reaction temperature with or without simultaneous cooling (CEM PowerMAX methodology). ${ }^{13}$ The best conditions gave conversions of $65 \%$ and these were obtained at $170{ }^{\circ} \mathrm{C}$ without external cooling. Higher temperatures produced a clear reduction in yield and a charred reaction mixture was obtained at $200{ }^{\circ} \mathrm{C}$. A series of $N$-aminides $2 \mathbf{e}-\mathbf{g}, \mathbf{2 i}-\mathbf{k}, \mathbf{2 0}-\mathbf{q}, \mathbf{2 s}, \mathbf{2 u}-\mathbf{v}, \mathbf{2 x}-\mathbf{z}$, and 2aa (see Table 1 , method $\mathrm{C}$ ) were synthesized according to this green methodology (Scheme 2), which reduces reaction times from hours (4-39 h) to $10 \mathrm{~min}$ without appreciable changes in yield.

The $N$-aminide 2a was also obtained by this method but, as expected due to the absence of electron-withdrawing substituents, the yield was considerably lower than that obtained previously by method B (see Table 1).

\section{Conclusions}

In an effort to expand an easy preparation of pyridinium $\mathrm{N}$-heteroarylaminides $\mathbf{2}$, three methods have been compared: one in which the aromatic substitution is produced using base and the conventional acetonitrile reflux (method $A$ ), a second approach using Pd catalysis (method B), and a third method using water as solvent, base, and microwave irradiation (method C). Method B is a new one-step amination procedure that provided the corresponding $\mathrm{N}$-aminides from haloheterocycles without electronwithdrawing substituents (see 2n) and even with the halogen located in positions that were not conjugated with nitrogen (see $\mathbf{2 m}, \mathbf{w}$ ). In most cases, however, only moderate yields were obtained. Finally, method $C$ is a green methodology that avoids the use of palladium and hard bases, with water used as a cheap and non-toxic solvent in conjunction with microwave heating, an approach that produces good yields and requires much shorter reaction times.

\section{Experimental}

\subsection{General remarks}

All melting points were determined in open capillary tubes on a Stuart Scientific SMP3 melting point apparatus and are uncorrected. IR spectra were obtained on a Perkin-Elmer FTIR spectrum 2000 spectrophotometer. ${ }^{1} \mathrm{H}$ NMR spectra were recorded on Varian Unity $300(300 \mathrm{MHz})$ and Varian Mercury-VX (500 MHz) spectrometers at ambient temperature. ${ }^{13} \mathrm{C}$ NMR spectra were recorded on Varian Unity $300(75 \mathrm{MHz})$ and Varian Mercury-VX (125 MHz) spectrometers at ambient temperature. Chemical shifts are given in parts per million $(\delta)$ downfield from TMS. Coupling constants $(J)$ are in hertz $(\mathrm{Hz})$ and signals are described as follows: s, singlet; d, doublet; t, triplet; m, multiplet; br, broad; ap, apparent. HPLC-MS analyses were performed on an Agilent 1100 apparatus. A Luna C18 $(150 \times 4.6 \mathrm{~mm}) 3 \mu \mathrm{m}$ Phenomenex chromatographic column was used with a mobile phase formed by a triple gradient of $4 \%$ aq formic acid (A), water (B), and acetonitrile (C). The gradient started as $A(2.5 \%), B(93 \%)$, and $C(4.5 \%)$ and in 30 min reached $A$ (2.5\%), B (4.5\%), and C (93\%). In the mass detector, the fragmenter operated at $70 \mathrm{eV}$. Low-resolution mass spectra (MS) were recorded on a Hewlett-Packard 5988A spectrometer using electronic impact (EI) or electrospray (ESI) and high-resolution analysis (TOF) was performed on an Agilent 6210 time-of-flight LC/MS. All reagents were obtained from commercial sources and were used without further purification. Solvents were purified and dried by standard procedures. Column chromatography was carried out with silica gel $60(40-63 \mu \mathrm{m}$, Merck) columns, using the eluent quoted in each case. All microwave experiments were carried out in a CEM Explorer PLS, a single mode operating system, working at 
$2.45 \mathrm{GHz}$, with a power programmable from 1 to $300 \mathrm{~W}$. Magnetic stirring was performed at $400 \mathrm{rpm}$, the only stirring speed allowed by the apparatus.

\subsection{General procedures for the preparation of pyridinium $\mathbf{N}$ - (heteroaryl) aminides (2)}

\subsubsection{Method A}

Potassium carbonate $(1.86 \mathrm{~g}, 13.5 \mathrm{mmol})$ was added to a solution of $\mathrm{N}$-aminopyridinium iodide $(1 \mathrm{~g}, 4.5 \mathrm{mmol})$ in acetonitrile $(20 \mathrm{~mL})$ and the reaction mixture was vigorously stirred for $45 \mathrm{~min}$ at room temperature to give a purple solution. To the reaction mixture was added a solution of the corresponding haloheterocycle $(4.7 \mathrm{mmol})$ in acetonitrile $(5 \mathrm{~mL})$. The mixture was stirred and heated under reflux until all starting material had been consumed (detected by TLC). The inorganic salts were filtered through Celite and the filtrate was evaporated in vacuo. The product was purified by chromatography on silica gel using ethanol as eluent. The solid was crystallized from the appropriate solvent and identified.

\subsubsection{Method $B$}

A round-bottomed flask was charged with heteroaryl halide $(0.37 \mathrm{mmol})$, sodium tert-butoxide $(0.22 \mathrm{~g}, 2.25 \mathrm{mmol})$, BINAP ( $5 \mathrm{~mol} \%$ ), and $N$-aminopyridinium iodide $(0.125 \mathrm{~g}, 0.56 \mathrm{mmol})$. The mixture was flushed with argon for $10 \mathrm{~min}$. Dry toluene $(10 \mathrm{~mL})$ and $\mathrm{Pd}_{2}(\mathrm{dba})_{3}(5 \mathrm{~mol} \%)$ were added. The mixture was stirred and heated under reflux until the starting material had been consumed (detected by TLC). The inorganic salts were filtered through Celite and the filtrate was evaporated in vacuo. The product was purified by chromatography on silica gel using ethanol as eluent. The solid was crystallized from the appropriate solvent and identified.

\subsubsection{Method C}

Potassium carbonate $(0.15 \mathrm{~g}, 1.12 \mathrm{mmol}), \mathrm{N}$-aminopyridinium iodide $(0.083 \mathrm{~g}, 0.37 \mathrm{mmol})$, the corresponding haloheterocycle $(0.39 \mathrm{mmol})$, and water $(0.5 \mathrm{~mL})$ were placed in a standard CEM $10 \mathrm{~mL}$ pressurized reaction vial. The reaction mixture was then magnetically stirred and irradiated in the microwave system at $170{ }^{\circ} \mathrm{C}$ for $10 \mathrm{~min}$. The solvent was removed under vacuum and the product was purified by chromatography on silica gel, using ethanol as eluent, with the exception of compounds $2 \mathbf{s}$ and $\mathbf{2 t}$, which precipitated from the reaction mixture and were recovered by filtration. Finally, the products were crystallized from the appropriate solvent and identified.

The following compounds were prepared according to one or more of the general methods described before.

\subsubsection{1. $N$-(Pyridin-2-yl)pyridinium aminide 2a. See Ref. $6 b$.}

4.2.3.2. $N$-(5-Methylpyridin-2-yl)pyridinium aminide $\mathbf{2 b}$. Orange solid, mp 94-96 ${ }^{\circ} \mathrm{C}$ (ethanol); IR $(\mathrm{KBr}) \nu_{\max }\left(\mathrm{cm}^{-1}\right)$ : 1599, 1463, 1374, 1320, 1285, 1147, 806, 669; ${ }^{1} \mathrm{H}$ NMR (300 MHz, CD $\left.{ }_{3} \mathrm{OD}\right): \delta 8.76$ $(2 \mathrm{H}, \mathrm{dd}, J=7.0$ and $1.3 \mathrm{~Hz}, \mathrm{H2}(6)), 7.99(1 \mathrm{H}, \mathrm{tt}, J=7.7$ and $1.3 \mathrm{~Hz}, \mathrm{H} 4)$, $7.79(2 \mathrm{H}, \mathrm{dd}, J=7.7$ and $7.0 \mathrm{~Hz}, H 3(5)), 7.55\left(1 \mathrm{H}, \mathrm{m}, H 6^{\prime}\right), 7.30(1 \mathrm{H}$, dd, $J=8.6$ and $\left.2.4 \mathrm{~Hz}, H 4^{\prime}\right), 6.52\left(1 \mathrm{H}, \mathrm{d}, J=8.6 \mathrm{~Hz}, \mathrm{H3}^{\prime}\right), 2.15\left(3 \mathrm{H}, \mathrm{s}, \mathrm{CH}_{3}\right)$; ${ }^{13} \mathrm{C}$ NMR (75 MHz, CD 3 OD): $\delta$ 163.4, 145.7, 144.0, 140.1, 137.1, 128.5, 121.8, 112.3, 17.5. MS (EI, $m / z): 185$ (31, $\left.\mathrm{M}^{+}\right), 184$ (86), 105 (11), 79 (44), 52 (100); HRMS (ESI-TOF, $\mathrm{CH}_{3} \mathrm{OH}$ ): calcd for $\mathrm{C}_{11} \mathrm{H}_{12} \mathrm{~N}_{3}[\mathrm{M}+\mathrm{H}]^{+}$ 186.10257; found, 186.10226 .

\subsubsection{3. $N$-(5-Bromopyridin-2-yl)pyridinium aminide $2 c$. See Ref. 6c.}

4.2.3.4. $\mathrm{N}$-(3-Methoxypyridin-2-yl)pyridinium aminide 2d. Orange solid, mp $181-183^{\circ} \mathrm{C}$ (ethanol); IR (KBr) $\nu_{\max }\left(\mathrm{cm}^{-1}\right)$ : 1593,1465 , 1442, 1420, 1209, 1179, 1109, 1003, 745; ${ }^{1} \mathrm{H}$ NMR (300 MHz, CD $\left.{ }_{3} \mathrm{OD}\right)$ : $\delta 8.72(2 \mathrm{H}$, dd, $J=6.9$ and $1.3 \mathrm{~Hz}, H 2(6)), 8.07(1 \mathrm{H}, \mathrm{tt}, J=7.7$ and
$1.3 \mathrm{~Hz}, H 4), 7.83(2 \mathrm{H}, \mathrm{dd}, J=7.7$ and $6.9 \mathrm{~Hz}, H 3(5)), 7.28(1 \mathrm{H}$, dd, $J=5.3$ and $\left.1.5 \mathrm{~Hz}, H 6^{\prime}\right), 6.96\left(1 \mathrm{H}, \mathrm{dd}, J=7.7\right.$ and $\left.1.5 \mathrm{~Hz}, H 4^{\prime}\right), 6.45(1 \mathrm{H}$, $\mathrm{dd}, J=7.7$ and $\left.5.3 \mathrm{~Hz}, \mathrm{H5}^{\prime}\right), 3.89\left(3 \mathrm{H}, \mathrm{s}, \mathrm{CH}_{3}\right) ;{ }^{13} \mathrm{C} \mathrm{NMR}(75 \mathrm{MHz}$, $\left.\mathrm{CD}_{3} \mathrm{OD}\right): \delta 157.4,145.4,145.1,138.3,137.5,128.5,115.4,112.1,55.6$. MS (EI, $m / z): 201$ (66, M+ $\left.{ }^{+}\right), 200$ (100), 107 (64), 80 (59), 67 (19), 53 (38), 52 (31); HRMS (ESI-TOF, $\mathrm{CH}_{3} \mathrm{OH}$ ): calcd for $\mathrm{C}_{11} \mathrm{H}_{12} \mathrm{~N}_{3} \mathrm{O}$ $[\mathrm{M}+\mathrm{H}]^{+}$, 202.09749; found, 202.09668.

4.2.3.5. N-(6-Chloropyridin-2-yl)pyridinium aminide $2 \boldsymbol{e}$. See Ref. 6j.

4.2.3.6. $N$-(5-Nitropyridin-2-yl)pyridinium aminide 2 f. See Ref. $6 b$.

4.2.3.7. N-(3-Nitropyridin-2-yl)pyridinium aminide 2g. See Ref. 6j.

4.2.3.8. $\mathrm{N}$-(6'-Bromo[2,2']bipyridin-6-yl)pyridinium aminide 2h. Yellow solid, mp $190-192{ }^{\circ} \mathrm{C}$ (ethanol/ethyl acetate); IR ( $\mathrm{KBr}$ ) $\nu_{\max }\left(\mathrm{cm}^{-1}\right)$ : $1570,1547,1431,1412,1353,1268,1237,1139,1124$, $980,784,666 ;{ }^{1} \mathrm{H}$ NMR $\left(300 \mathrm{MHz}, \mathrm{CD}_{3} \mathrm{OD}\right): \delta 8.93(2 \mathrm{H}, \mathrm{dd}, J=6.9$ and $1.3 \mathrm{~Hz}, \mathrm{H2}(6)), 8.06$ ( $1 \mathrm{H}, \mathrm{tt}, J=7.6$ and $1.3 \mathrm{~Hz}, \mathrm{H} 4), 7.85(2 \mathrm{H}, \mathrm{dd}, J=7.6$ and $6.9 \mathrm{~Hz}, H 3(5)), 7.84\left(1 \mathrm{H}, \mathrm{dd}, J=7.6\right.$ and $\left.1.0 \mathrm{~Hz}, H 3^{\prime \prime}\right), 7.59(1 \mathrm{H}, \mathrm{t}$, $\left.J=7.7 \mathrm{~Hz}, H 4^{\prime \prime}\right), 7.48\left(3 \mathrm{H}, \mathrm{m}, H 3^{\prime}, H 4^{\prime}\right.$ and $\left.H 5^{\prime \prime}\right), 6.60(1 \mathrm{H}, \mathrm{dd}, J=8.1$ and $\left.1.1 \mathrm{~Hz}, H 5^{\prime}\right) ;{ }^{13} \mathrm{C}$ NMR (75 MHz, $\left.\mathrm{CD}_{3} \mathrm{OD}\right): \delta 164.5,159.6,152.1$, $144.6,142.2,140.4,138.8,137.6,128.2,127.9,120.1,113.0,110.7$. MS (EI, $m / z): 326 / 328\left(50 / 51, \mathrm{M}^{+}\right), 325 / 327$ (90/100), 249/251 (13/12), 246 (48), 194 (16), 170 (43), 141 (29), 114 (26), 79 (49), 52 (36); HRMS (ESI-TOF, $\mathrm{CH}_{3} \mathrm{OH}$ ): calcd for $\mathrm{C}_{15} \mathrm{H}_{12}^{79} \mathrm{BrN}_{4}[\mathrm{M}+\mathrm{H}]^{+}, 327.0245$; found, 327.0250 .

\subsubsection{9. $N$-(Quinolin-2-yl)pyridinium aminide $2 \boldsymbol{i}$. See Ref. 6b.}

4.2.3.10. $\mathrm{N}$-(Quinolin-4-yl)pyridinium aminide $\mathbf{2 j}$. Orange solid, mp 93-95 ${ }^{\circ} \mathrm{C}$ (ethanol/ethyl acetate); IR $(\mathrm{KBr}) \nu_{\max }\left(\mathrm{cm}^{-1}\right): 1647,1618$, 1601, 1539, 1494, 1468, 1389, 1354, 1136, 789, 745, 674; ${ }^{1} \mathrm{H}$ NMR ( $300 \mathrm{MHz}, \mathrm{CD}_{3} \mathrm{OD}$ ): $\delta 8.85$ (2H, dd, $J=6.7$ and $1.4 \mathrm{~Hz}, H 2(6)$ ), 8.47 $\left(1 \mathrm{H}, \mathrm{br} \mathrm{d}, J=8.2 \mathrm{~Hz}, H 8^{\prime}\right), 8.36(1 \mathrm{H}, \mathrm{tt}, J=7.8$ and $1.4 \mathrm{~Hz}, H 4), 8.06(2 \mathrm{H}$, dd, $J=7.8$ and $6.7 \mathrm{~Hz}, H 3(5)), 7.68(1 \mathrm{H}$, ddd, $J=8.1,6.8$, and $1.4 \mathrm{~Hz}$, $\left.H 7^{\prime}\right), 7.62\left(1 \mathrm{H}, \mathrm{dd}, J=8.1\right.$ and $\left.1.6 \mathrm{~Hz}, H 5^{\prime}\right), 7.51(1 \mathrm{H}$, ddd, $J=8.3,6.8$, and $\left.1.2 \mathrm{~Hz}, H 6^{\prime}\right), 7.25\left(1 \mathrm{H}, \mathrm{d}, J=6.6 \mathrm{~Hz}, H 2^{\prime}\right), 6.71(1 \mathrm{H}, \mathrm{d}, J=6.6 \mathrm{~Hz}$, $\left.H 3^{\prime}\right) ;{ }^{13} \mathrm{C}$ NMR (75 MHz, CD $\mathrm{OD}$ ): $\delta$ 145.7, 141.5, 138.3, 134.8, 132.5, $129.9,129.8,127.5,127.2,126.2,122.4,109.4$. MS (ESI, $m / z): 222$ (100, $\mathrm{M}+1$ ), 220 (28), 143 (18); HRMS (ESI-TOF, $\mathrm{CH}_{3} \mathrm{OH}$ ): calcd for $\mathrm{C}_{14} \mathrm{H}_{12} \mathrm{~N}_{3}[\mathrm{M}+\mathrm{H}]^{+}, 222.1031$; found, 222.1044 .

4.2.3.11. $\mathrm{N}$-(Isoquinolin-1-yl)pyridinium aminide $\mathbf{2 k}$. Orange solid, mp 138-140 ${ }^{\circ} \mathrm{C}$ (ethanol/ethyl acetate); IR $(\mathrm{KBr}) \nu_{\max }\left(\mathrm{cm}^{-1}\right): 1612$, 1583, 1540, 1495, 1472, 1391, 1356, 1157, 1136, 789, 750, 676; ${ }^{1} \mathrm{H}$ NMR (300 MHz, CD $30 D$ ): $\delta 8.83(2 \mathrm{H}, \mathrm{dd}, J=6.9$ and $1.3 \mathrm{~Hz}, \mathrm{H2}(6)$ ), $8.44\left(1 \mathrm{H}, \mathrm{br} \mathrm{dd}, J=8.2\right.$ and $\left.0.9 \mathrm{~Hz}, H 8^{\prime}\right), 8.30(1 \mathrm{H}, \mathrm{tt}, J=7.8$ and $1.3 \mathrm{~Hz}$, $H 4), 8.02(2 \mathrm{H}, \mathrm{dd}, J=7.8$ and $6.9 \mathrm{~Hz}, H 3(5)), 7.65(1 \mathrm{H}, \mathrm{ddd}, J=7.9,6.4$, and $\left.1.3 \mathrm{~Hz}, H 6^{\prime}\right), 7.60\left(1 \mathrm{H}\right.$, br dd, $J=7.9$ and $\left.2.0 \mathrm{~Hz}, H 5^{\prime}\right), 7.49(1 \mathrm{H}$, ddd, $J=8.2,6.4$, and $\left.1.8 \mathrm{~Hz}, H 7^{\prime}\right), 7.32\left(1 \mathrm{H}, \mathrm{d}, J=6.4 \mathrm{~Hz}, H 3^{\prime}\right), 6.71(1 \mathrm{H}$, br d, $\left.J=6.4 \mathrm{~Hz}, H 4^{\prime}\right) ;{ }^{13} \mathrm{C} \mathrm{NMR}\left(75 \mathrm{MHz}, \mathrm{CD}_{3} \mathrm{OD}\right): \delta 162.8,146.1,140.3$, 138.8, 138.5, 131.6, 129.3, 126.9, 126.7, 125.9, 122.0, 109.4. MS (ESI, m/z): 222 (100, $\mathrm{M}+1$ ), 220 (9), 143 (24); HRMS (ESI-TOF, $\mathrm{CH}_{3} \mathrm{OH}$ ): calcd for $\mathrm{C}_{14} \mathrm{H}_{12} \mathrm{~N}_{3}[\mathrm{M}+\mathrm{H}]^{+}, 222.1031$; found, 222.1045 .

4.2.3.12. $\mathrm{N}$-(3-Chloroisoquinolin-1-yl)pyridinium aminide 2l. Orange solid, mp $133-135^{\circ} \mathrm{C}$ (ethanol/ethyl acetate); IR (KBr) $\nu_{\max }\left(\mathrm{cm}^{-1}\right): 1579,1486,1474,1407,1381,1139,671 ;{ }^{1} \mathrm{H}$ NMR (300 MHz, CD $\left.{ }_{3} \mathrm{OD}\right): \delta 8.84(2 \mathrm{H}, \mathrm{dd}, J=7.0$ and $1.3 \mathrm{~Hz}, \mathrm{H} 2(6)), 8.33$ $\left(1 \mathrm{H}, \mathrm{br} \mathrm{dd}, J=8.4\right.$ and $\left.1.2 \mathrm{~Hz}, H 8^{\prime}\right), 8.21(1 \mathrm{H}, \mathrm{tt}, J=7.8$ and $1.3 \mathrm{~Hz}, H 4)$, $7.94(2 \mathrm{H}, \mathrm{dd}, J=7.8$ and $7.0 \mathrm{~Hz}, H 3(5)), 7.56(1 \mathrm{H}$, ddd, $J=8.1,6.8$, and $\left.1.2 \mathrm{~Hz}, H 6^{\prime}\right), 7.49\left(1 \mathrm{H}, \mathrm{dd}, J=8.1\right.$ and $\left.1.5 \mathrm{~Hz}, H 5^{\prime}\right), 7.39(1 \mathrm{H}$, ddd, $J=8.4,6.7$, and $\left.1.5 \mathrm{~Hz}, H 7^{\prime}\right), 6.66\left(1 \mathrm{H}, \mathrm{s}, H 4^{\prime}\right) ;{ }^{13} \mathrm{C} \mathrm{NMR}(75 \mathrm{MHz}$, $\left.\mathrm{CD}_{3} \mathrm{OD}\right): \delta 163.5,146.0,145.4,140.6,139.8,131.4,128.6,126.1,126.0$, 125.7, 120.1, 106.2. MS (ESI, $m / z):$ 256/258 (100/36, M+1), 177 (25); 
HRMS (APCI): calcd for $\mathrm{C}_{14} \mathrm{H}_{11}^{35} \mathrm{ClN}_{3}[\mathrm{M}+\mathrm{H}]^{+}, 256.0642$; found, 256.0641.

4.2.3.13. $\mathrm{N}$-(Isoquinolin-4-yl)pyridinium aminide $\mathbf{2 m}$. Dark red solid, mp $159-161^{\circ} \mathrm{C}$ (ethanol/ethyl acetate); IR $(\mathrm{KBr}) \nu_{\max }\left(\mathrm{cm}^{-1}\right)$ : 1558, 1489, 1465, 1457, 1396, 1320, 1124, 678, 668; ${ }^{1} \mathrm{H}$ NMR (300 MHz, CD $\left.{ }_{3} \mathrm{OD}\right): \delta 8.83(2 \mathrm{H}, \mathrm{dd}, J=7.0$ and $1.3 \mathrm{~Hz}, \mathrm{H} 2(6)), 8.50$ $\left(1 \mathrm{H}, \mathrm{s}, H 1^{\prime}\right), 8.31\left(1 \mathrm{H}, \mathrm{m}, H 8^{\prime}\right), 8.14(1 \mathrm{H}, \mathrm{tt}, J=7.8$ and $1.3 \mathrm{~Hz}, \mathrm{H} 4), 7.96$ (1H, m, H5'), $7.91(2 \mathrm{H}, \mathrm{dd}, J=7.8$ and $7.0 \mathrm{~Hz}, H 3(5)), 7.69\left(2 \mathrm{H}, \mathrm{m}, H 6^{\prime}\right.$ and $\left.H 7^{\prime}\right), 7.29\left(1 \mathrm{H}, \mathrm{s}, H 3^{\prime}\right) ;{ }^{13} \mathrm{CNMR}\left(75 \mathrm{MHz}, \mathrm{CD}_{3} \mathrm{OD}\right): \delta$ 146.2, 145.7, 143.2, 142.2, 131.9, 130.3, 129.6, 129.1, 129.0, 125.9 (two overlapped signals), 122.5. MS (EI, $m / z): 221\left(15, \mathrm{M}^{+}\right), 144$ (19), 142 (24), 115 (40), 80 (65), 79 (100), 52 (80); HRMS (ESI-TOF, $\mathrm{CH}_{3} \mathrm{OH}$ ): calcd for $\mathrm{C}_{14} \mathrm{H}_{12} \mathrm{~N}_{3}[\mathrm{M}+\mathrm{H}]^{+}, 222.1031$; found, 222.1022 .

4.2.3.14. N-(6-Methoxypyridazin-3-yl)pyridinium aminide 2n. Orange solid, mp $115-117^{\circ} \mathrm{C}$ (ethanol); IR $(\mathrm{KBr}) \nu_{\max }\left(\mathrm{cm}^{-1}\right)$ : $1559,1472,1418,1304,1006,841,667 ;{ }^{1} \mathrm{H}$ NMR (300 MHz, CD ${ }_{3} \mathrm{OD}$ ): $\delta 8.90(2 \mathrm{H}, \mathrm{dd}, J=7.0$ and $1.3 \mathrm{~Hz}, H 2(6)), 8.33(1 \mathrm{H}, \mathrm{tt}, J=7.8$ and $1.3 \mathrm{~Hz}, \mathrm{H} 4), 8.03(2 \mathrm{H}, \mathrm{dd}, J=7.8$ and $7.0 \mathrm{~Hz}, \mathrm{H3}(5)), 7.09(1 \mathrm{H}$, ap d, $J=9.7 \mathrm{~Hz}, H 4^{\prime}$ or $\left.H 5^{\prime}\right), 7.02\left(1 \mathrm{H}\right.$, ap d, $J=9.7 \mathrm{~Hz}, H 5^{\prime}$ or $\left.H 4^{\prime}\right), 3.88(3 \mathrm{H}$, $\left.\mathrm{S}, \mathrm{CH}_{3}\right) ;{ }^{13} \mathrm{C}$ NMR $\left(75 \mathrm{MHz}, \mathrm{CD}_{3} \mathrm{OD}\right): \delta 163.2,160.2,145.1,138.5$, 128.6, 125.0, 121.6, 54.3. MS (EI, $m / z): 202\left(32, \mathrm{M}^{+}\right), 201(100), 80$ (74), 79 (16), 52 (39); HRMS (ESI-TOF, $\mathrm{CH}_{3} \mathrm{OH}$ ): calcd for $\mathrm{C}_{10} \mathrm{H}_{11} \mathrm{~N}_{4} \mathrm{O}$ $[\mathrm{M}+\mathrm{H}]^{+}$, 203.0933; found, 203.0929.

4.2.3.15. N-(6-Chloropyridazin-2-yl)pyridinium aminide 2o. See Ref. $6 \mathrm{j}$.

4.2.3.16. $N$-(6-Phenylpyridazin-2-yl)pyridinium aminide $2 \boldsymbol{p}$. See Ref. $6 \mathrm{j}$.

4.2.3.17. $N$-(Pyrimidin-2-yl)pyridinium aminide 2q. See Ref. 6b,j.

4.2.3.18. $N$-(5-Bromopyrimidin-2-yl)pyridinium aminide 2r. Yellow solid, mp $126-127^{\circ} \mathrm{C}$ (dichloromethane/diethyl ether); IR (KBr) $\nu_{\max }\left(\mathrm{cm}^{-1}\right): 1575,1507,1473,1436,1267,781,669 ;{ }^{1} \mathrm{H}$ NMR (300 MHz, CD $\left.{ }_{3} \mathrm{OD}\right): \delta 8.75(2 \mathrm{H}, \mathrm{dd}, J=6.9$ and $1.3 \mathrm{~Hz}, \mathrm{H2}(6)), 8.23$ $(1 \mathrm{H}, \mathrm{tt}, J=7.8$ and $1.3 \mathrm{~Hz}, H 4), 8.11\left(2 \mathrm{H}, \mathrm{s}, H 4^{\prime}\left(6^{\prime}\right)\right), 7.93(2 \mathrm{H}, \mathrm{dd}, J=7.8$ and $6.9 \mathrm{~Hz}, \mathrm{H3}(5)) ;{ }^{13} \mathrm{C} \mathrm{NMR}\left(75 \mathrm{MHz}, \mathrm{CD}_{3} \mathrm{OD}\right): \delta 167.4,159.2,145.8$, 140.5, 128.7, 103.8. MS (EI, $m / z): 252 / 250\left(38 / 38, \mathrm{M}^{+}\right), 251 / 249$ (100/97), 119 (26), 79 (90), 52 (29); HRMS (ESI-TOF, $\mathrm{CH}_{3} \mathrm{OH}$ ): calcd for $\mathrm{C}_{9} \mathrm{H}_{8}^{79} \mathrm{BrN}_{4}[\mathrm{M}+\mathrm{H}]^{+}$, 250.9932; found, 250.9933 .

4.2.3.19. N-(2-Chloropyrimidin-4-yl)pyridinium aminide 2 s. See Ref. 6j.

4.2.3.20. $N$-(2-Methylsulfanylpyrimidin-4-yl)pyridinium aminide 2t. Orange solid, $\mathrm{mp} 70-71{ }^{\circ} \mathrm{C}$ (ethyl acetate/hexane); IR (KBr) $\nu_{\max }$ $\left(\mathrm{cm}^{-1}\right): 1618,1582,1502,1473,1449,1368,1353,813,687 ;{ }^{1} \mathrm{H}$ NMR (300 MHz, CD $\left.{ }_{3} \mathrm{OD}\right): \delta 8.72(2 \mathrm{H}, \mathrm{dd}, J=6.9$ and $1.3 \mathrm{~Hz}, \mathrm{H} 2(6)), 8.28$ $(1 \mathrm{H}, \mathrm{tt}, J=7.8$ and $1.3 \mathrm{~Hz}, \mathrm{H} 4), 7.95(2 \mathrm{H}, \mathrm{dd}, J=7.8$ and $6.9 \mathrm{~Hz}, \mathrm{H} 3(5))$, $7.66\left(1 \mathrm{H}, \mathrm{d}, J=6.2 \mathrm{~Hz}, H 6^{\prime}\right), 6.09\left(1 \mathrm{H}, \mathrm{d}, J=6.2 \mathrm{~Hz}, H 5^{\prime}\right), 2.07(3 \mathrm{H}, \mathrm{s}$, $\left.\mathrm{CH}_{3}\right) ;{ }^{13} \mathrm{C}$ NMR $\left(75 \mathrm{MHz}, \mathrm{CD}_{3} \mathrm{OD}\right): \delta 171.3,167.5,153.2,146.1,140.9$, 128.4, 102.6, 13.5. MS (EI, $m / z): 218\left(52, \mathrm{M}^{+}\right), 217$ (26), 80 (33), 79 (100), 52 (27); HRMS (ESI-TOF, $\mathrm{CH}_{3} \mathrm{OH}$ ): calcd for $\mathrm{C}_{10} \mathrm{H}_{11} \mathrm{~N}_{4} \mathrm{~S}$ $[\mathrm{M}+\mathrm{H}]^{+}$, 219.0704; found, 219.0721.

4.2.3.21. N-(2-Chloro-6 methylpyrimidin-4-yl)pyridinium aminide 2u. See Ref. 6j.

4.2.3.22. $N$-(2,6-Dichloropyrimidin-4-yl)pyridinium aminide $2 \boldsymbol{v}$. See Ref. $6 \mathrm{j}$.

4.2.3.23. $N$-(Pyrimidin-5-yl)pyridinium aminide $2 \boldsymbol{w}$. Orange solid, mp 131-133 ${ }^{\circ} \mathrm{C}$ (ethanol/ethyl acetate); IR $(\mathrm{KBr}) \nu_{\max }\left(\mathrm{cm}^{-1}\right)$ : 1458 ,
1431, 719, 626; ${ }^{1} \mathrm{H}$ NMR (300 MHz, CD $30 D$ ): $\delta 8.81$ (2H, dd, $J=6.9$ and $1.2 \mathrm{~Hz}, H 2(6)), 8.23\left(1 \mathrm{H}, \mathrm{s}, H 2^{\prime}\right), 8.16(1 \mathrm{H}, \mathrm{tt}, J=7.6$ and $1.2 \mathrm{~Hz}$, $H 4), 7.92\left(4 \mathrm{H}, \mathrm{m}, H 4^{\prime}\left(6^{\prime}\right)\right.$ and $\left.H 3(5)\right) ;{ }^{13} \mathrm{C}$ NMR (75 MHz, $\left.\mathrm{CD}_{3} \mathrm{OD}\right)$ : $\delta 151.9,145.3,144.2,140.4,139.0,130.0 . \mathrm{MS}(\mathrm{EI}, m / z): 172\left(48, \mathrm{M}^{+}\right)$, 80 (99), 66 (100), 52 (40); HRMS (ESI-TOF, $\mathrm{CH}_{3} \mathrm{OH}$ ): calcd for $\mathrm{C}_{9} \mathrm{H}_{9} \mathrm{~N}_{4}[\mathrm{M}+\mathrm{H}]^{+}, 173.0827$; found, 173.0824 .

4.2.3.24. N-(Pyrazin-2-yl)pyridinium aminide $2 \boldsymbol{x}$. See Ref. 6b,j.

4.2.3.25. N-(3-Chloropyrazin-2-yl)pyridinium aminide 2y. See Ref. 6j.

4.2.3.26. N-(6-Chloropyrazin-2-yl)pyridinium aminide $2 \boldsymbol{z}$. See Ref. 6j.

4.2.3.27. N-(Quinoxalin-2-yl)pyridinium aminide 2aa. Yellow solid, $\mathrm{mp} 132-134{ }^{\circ} \mathrm{C}$ (ethanol/ethyl acetate); IR $(\mathrm{KBr}) \nu_{\max }\left(\mathrm{cm}^{-1}\right): 1580$, 1541, 1503, 1467, 1430, 1304, 1153, 1011, 928, 754, 666; ${ }^{1} \mathrm{H}$ NMR (300 MHz, CD ${ }_{3} \mathrm{OD}$ ): $\delta 8.97$ ( $2 \mathrm{H}$, dd, $J=7.0$ and $1.3 \mathrm{~Hz}, H 2(6)$ ), 8.23 $(1 \mathrm{H}, \mathrm{tt}, J=7.9$ and $1.3 \mathrm{~Hz}, H 4), 8.21\left(1 \mathrm{H}, \mathrm{s}, H 3^{\prime}\right), 7.96(2 \mathrm{H}, \mathrm{dd}, J=7.9$ and $7.0 \mathrm{~Hz}, H 3(5)), 7.68\left(1 \mathrm{H}, \mathrm{dd}, J=8.2\right.$ and $\left.1.5 \mathrm{~Hz}, H 5^{\prime}\right), 7.42(1 \mathrm{H}$, ddd, $J=8.2,7.0$, and $\left.1.5 \mathrm{~Hz}, H 6^{\prime}\right), 7.28\left(1 \mathrm{H}, \mathrm{dd}, J=8.2\right.$ and $\left.1.5 \mathrm{~Hz}, H 8^{\prime}\right)$, 7.21 (ddd, $J=8.2,7.0$, and $1.5 \mathrm{~Hz}, H 7^{\prime}$ ); ${ }^{13} \mathrm{C}$ NMR (75 $\mathrm{MHz}, \mathrm{CD}_{3} \mathrm{OD}$ ): $\delta$ 159.6, 145.0, 144.7, 143.3, 139.8, 137.1, 130.7, 128.7, 128.6, 125.4, 123.5. MS (EI, $m / z): 222\left(50, \mathrm{M}^{+}\right), 221$ (100), 143 (15), 116 (28), 79 (12), 52 (12); HRMS (APCI): calcd for $\mathrm{C}_{13} \mathrm{H}_{11} \mathrm{~N}_{4}[\mathrm{M}+\mathrm{H}]^{+}, 223.0984$; found, 223.1041.

4.2.3.28. $\mathrm{N}$-(Thiazol-2-yl)pyridinium aminide $\mathbf{2 a b}$. Orange solid, $\mathrm{mp}$ 95-97 ${ }^{\circ} \mathrm{C}$ (ethyl acetate/hexane); IR (KBr) $\nu_{\max }\left(\mathrm{cm}^{-1}\right): 1491,1444$, 1309, 1223, 1162, 757, 667; ${ }^{1} \mathrm{H}$ NMR (300 MHz, CD $\left.{ }_{3} \mathrm{OD}\right): \delta 9.10(2 \mathrm{H}$, $\mathrm{dd}, J=7.2$ and $1.3 \mathrm{~Hz}, H 2(6)), 7.97(1 \mathrm{H}, \mathrm{tt}, J=7.6$ and $1.3 \mathrm{~Hz}, H 4), 7.79$ $(2 \mathrm{H}, \mathrm{dd}, J=7.6$ and $7.2 \mathrm{~Hz}, H 3(5)), 6.93\left(1 \mathrm{H}, \mathrm{d}, J=3.8 \mathrm{~Hz}, H 4^{\prime}\right), 6.50$ $\left(1 \mathrm{H}, \mathrm{d}, J=3.8 \mathrm{~Hz}, \mathrm{H}^{\prime}\right) ;{ }^{13} \mathrm{C} \mathrm{NMR}\left(75 \mathrm{MHz},\left(\mathrm{CD}_{3}\right)_{2} \mathrm{CO}\right): \delta 178.8,141.7$, 138.2, 136.2, 128.3, 106.4. MS (EI, $m / z): 177\left(33, \mathrm{M}^{+}\right), 79$ (100), 52 (19); HRMS (ESI-TOF, $\mathrm{CH}_{3} \mathrm{OH}$ ): calcd for $\mathrm{C}_{8} \mathrm{H}_{8} \mathrm{~N}_{3} \mathrm{~S}[\mathrm{M}+\mathrm{H}]^{+}$, 178.0439; found, 178.0428 .

4.2.3.29. N-(5-Nitrothiazol-2-yl)pyridinium aminide 2ac. Orange solid, mp $241-242{ }^{\circ} \mathrm{C}$ (methanol); IR $(\mathrm{KBr}) \nu_{\max }\left(\mathrm{cm}^{-1}\right): 1505,1485$, 1473, 1417, 1388, 1317, 1238, 1159, 1110, 750, 667; ${ }^{1} \mathrm{H}$ NMR (300 MHz, $\left.\left(\mathrm{CD}_{3}\right)_{2} \mathrm{CO}\right): \delta 9.04(2 \mathrm{H}, \mathrm{dd}, J=7.0$ and $1.3 \mathrm{~Hz}, \mathrm{H} 2(6)), 8.33$ $(1 \mathrm{H}, \mathrm{tt}, J=7.7$ and $1.3 \mathrm{~Hz}, \mathrm{H} 4), 8.06(2 \mathrm{H}, \mathrm{dd}, J=7.7$ and $7.0 \mathrm{~Hz}, \mathrm{H3}(5))$, $7.94\left(1 \mathrm{H}, \mathrm{s}, \mathrm{H}^{\prime}\right) ;{ }^{13} \mathrm{C}$ NMR (125 MHz, $\left.\left(\mathrm{CD}_{3}\right)_{2} \mathrm{CO}\right): 179.5,142.5,139.5$, 133.0, 127.5 (two overlapped signals). MS (EI, $m / z): 222\left(37, \mathrm{M}^{+}\right), 97$ (35), 79 (100), 52 (27); HRMS (ESI-TOF, $\mathrm{CH}_{3} \mathrm{OH}$ ): calcd for $\mathrm{C}_{8} \mathrm{H}_{7} \mathrm{~N}_{4} \mathrm{O}_{2} \mathrm{~S}[\mathrm{M}+\mathrm{H}]^{+}$, 223.0290; found, 223.0277.

4.2.3.30. $\mathrm{N}$-(5-Ethoxycarbonylthiazol-2-yl)pyridinium aminide 2ad. Orange solid, $\mathrm{mp} 125-126^{\circ} \mathrm{C}$ (ethyl acetate/hexane); IR ( $\mathrm{KBr}$ ) $\nu_{\max }\left(\mathrm{cm}^{-1}\right): 1684,1489,1452,1311,1276,1234,1164,1074,770,749$, $670 ;{ }^{1} \mathrm{H}$ NMR (300 MHz, CD $\left.\mathrm{OD}\right): \delta 8.96(2 \mathrm{H}$, dd, $J=7.0$ and $1.3 \mathrm{~Hz}$, $H 2(6)), 8.21(1 \mathrm{H}, \mathrm{tt}, J=7.8$ and $1.3 \mathrm{~Hz}, H 4), 7.94(2 \mathrm{H}, \mathrm{dd}, J=7.8$ and $7.0 \mathrm{~Hz}, \mathrm{H} 3(5)), 7.62\left(1 \mathrm{H}, \mathrm{s}, H 4^{\prime}\right), 4.26\left(2 \mathrm{H}, \mathrm{q}, J=7.1 \mathrm{~Hz}, \mathrm{CH}_{2}\right), 1.34(3 \mathrm{H}$, $\left.\mathrm{t}, J=7.1 \mathrm{~Hz}, \mathrm{CH}_{3}\right) ;{ }^{13} \mathrm{C}$ NMR $\left(75 \mathrm{MHz}, \mathrm{CD}_{3} \mathrm{OD}\right): \delta 183.0,164.4,148.5$, 144.0, 140.1, 129.0, 113.4, 61.6, 14.7. MS (EI, $m / z): 249\left(34, \mathrm{M}^{+}\right), 97$ (22), 79 (100), 52 (38); HRMS (ESI-TOF, $\mathrm{CH}_{3} \mathrm{OH}$ ): calcd for $\mathrm{C}_{11} \mathrm{H}_{12} \mathrm{~N}_{3} \mathrm{O}_{2} \mathrm{~S}[\mathrm{M}+\mathrm{H}]^{+}, 250.0650$; found, 250.0671.

\section{Acknowledgements}

The authors wish to thank the Comision Interministerial de Ciencia y Tecnología (CICYT-BQU2001-1508 and CTQ2005-08902) for financial support and the Universidad de Alcalá (UAH) for a studentship (M.C.). 


\section{References and notes}

1. Ollis, D. W.; Stanforth, S. P. Tetrahedron 1985, 41, 2239-2329.

2. Britto, M. M.; Almeida, T. M. G.; Leitão, A.; Donnici, C. L.; Lopes, M. T. P. Montanari, C. A. Synth. Commun. 2006, 36, 3359-3369 and references cited therein.

3. (a) Senff-Ribeiro, A.; Echevarria, A.; Silva, E. F.; Franco, C. R. C.; Veiga, S. S Oliveira, M. B. M. Br. J. Cancer 2004, 91, 297-304; (b) da Silva, E. F.; CantoCavalheiro, M. M.; Braz, V. R.; Cysne-Finkelstein, L.; Leon, L. L.; Echevarria, A Eur. J. Med. Chem. 2002, 37, 979-984.

4. 1,3-Dipolar Cycloaddition Chemistry; Padwa, A., Ed.; Wiley-Interscience: New York, NY, 1984

5. (a) Vaquero, J. J.; Alvarez-Builla, J. Advances in Nitrogen Heterocycles; 2000; Vol. 4, pp 159-250; (b) Valenciano, J.; Cuadro, A. M.; Vaquero, J. J.; Alvarez-Builla, J. Tetrahedron Lett. 1999, 40, 763-766; (c) Crabb, D. L.; McCullough, K. J.; Preston, P. N.; Rosair, G. M.; Bishop, B. C.; Wright, S. H. B.; Clegg, W.; Coles, S. J. Chem. Soc., Perkin Trans. 1 1999, 1517-1526; (d) Bishop, B. C.; Marley, H.; Preston, P. N.; Wright, S. H. B. J. Chem. Soc., Perkin Trans. 1 1999, 1527-1532; (e) Schmidt, A.; Martin, N. Heterocycles 2001, 55, 827-834; (f) Butler, N. R.; Wallace, L. M. J. Chem. Soc., Perkin Trans. 1 2001, 1778-1784; (g) Valenciano, J.; Sanchez-Pavon, E.; Cuadro, A. M.; Vaquero, J. J.; Alvarez-Builla, J. J. Org. Chem. 2001, 66, 85288536; (h) Schmidt, A. J. Heterocycl. Chem. 2002, 39, 949-956; (i) Kappel, J. C.; Yokum, T. S.; Barany, G. J. Comb. Chem. 2004, 6, 746-752; (j) Dietrich, M.; Matthias, N. Heterocycles 2004, 63, 2605-2614; (k) Butler, R. N.; Fahy, A. M.; Fox A.; Stephens, J. C.; Cunningham, D.; Ryder, A. J. Org. Chem. 2006, 71, 5679-5687; (1) Moderhack, D.; Noreiks, M. Heterocycles 2006, 68, 2113-2122; (m) Valenciano, J.; Sanchez-Pavon, E.; Cuadro, A. M.; Alvarez-Builla, J.; Vaquero, J. J. Eur. J. Org. Chem. 2007, 15, 2423-2429.

6. (a) Carceller, R.; García-Navío, J. L.; Izquierdo, M. L.; Alvarez-Builla, J. Tetrahedron Lett. 1993, 34, 2019-2020; (b) Carceller, R.; García-Navío, J. L.; Izquierdo M. L.; Alvarez-Builla, J.; Fajardo, M.; Gómez-Sal, P.; Gago, F. Tetrahedron 1994 50, 4995-5012; (c) Burgos, C; Delgado, F. García-Navío, J. L.; Izquierdo, M. L Alvarez-Builla, J. Tetrahedron 1995, 51, 8649-8654; (d) García de Viedma, A Martinez-Barrasa, V.; Burgos, C.; Izquierdo, M. L.; Alvarez-Builla, J. J. Org. Chem. 1999, 64, 1007-1010; (e) Martínez-Barrasa, V.; Delgado, F.; Burgos, C.; GarcíaNavío, J. L.; Izquierdo, M. L.; Alvarez-Builla, J. Tetrahedron 2000, 56, 2481-2490; (f) Matínez-Barrasa, V.; García de Viedma, A.; Burgos, C.; Alvarez-Builla, J. Org. Lett. 2000, 2, 3933-3935; (g) De la Rosa, R.; Martinez-Barrasa, V.; Burgos, C. Alvarez-Builla, J. Tetrahedron Lett. 2000, 41, 5837-5840; (h) Nuñez, A.; García de Viedma, A.; Martínez-Barrasa, V.; Burgos, C.; Alvarez-Builla, J. Synlett 2002, 1093-1096; (i) Reyes, M. J.; Delgado, F.; Izquierdo, M. L.; Alvarez-Builla, J. Tetrahedron 2002, 58, 8573-8579; (j) Reyes, M. J: Burgos, C. Izquierdo, M. L; Alvarez-Builla, J. Tetrahedron 2004, 60, 1093-1097; (k) Reyes, M. J.; Izquierdo, M. L.; Alvarez-Builla, J. Tetrahedron Lett. 2004, 45, 8713-8715; (l) Nuñez, A.; Sánchez, A.; Burgos, C.; Alvarez-Builla, J. Tetrahedron 2004, 60, 6217-6224; (m) Sánchez, A.; Nuñez, A.; Alvarez-Builla, J:; Burgos, C. Tetrahedron 2004, 60 , 11843-11850; (n) Reyes, M. J.; Castillo, R.; Izquierdo, M. L.; Alvarez-Builla, J. Tetrahedron Lett. 2006, 47, 6457-6460; (o) Sánchez, A.; Nuñez, A.; Burgos, C.; Alvarez-Builla, J. Tetrahedron Lett. 2006, 47, 8343-8346; (p) Nuñez, A.; Sánchez, A.; Burgos, C.; Alvarez-Builla, J. Tetrahedron 2007, 63, 6774-6783; (q) Castillo, R.; Izquierdo, M. L.; Alvarez-Builla, J. Tetrahedron Lett. 2007, 48, 5899-5903; (r) Castillo, R.; Reyes, M. J.; Izquierdo, M. L.; Alvarez-Builla, J. Tetrahedron 2008, 64, 1351-1370.

7. Beyer, H.; Thieme, E. J. Prakt. Chem. 1966, 31, 293-303.

8. (a) Prim, D.; Campagne, J.-M.; Joseph, D.; Andrioletti, B. Tetrahedron 2002, 58, 2041-2075; (b) Kienle, M.; Dubbaka, S. R.; Brade, K.; Knochel, P. Eur. J. Org. Chem. 2007, 4166-4176 and references therein.

9. (a) Yang, B. H.; Buchwald, S. L. J. Organomet. Chem. 1999, 576, 125-146; (b) Strieter, E. R.; Blackmond, D. G.; Buchwald, S. L. J. Am. Chem. Soc. 2003, 125, 13978-13980

10. (a) Košmrly, J.; Maes, B. U. W.; Lemière, G. L. F.; Haemers, A. Synlett 2000, 1581 1584; (b) Meyers, C.; Maes, B. U. W.; Loones, K. T. J.; Bal, G.; Lemière, G. L. F.; Dommise, R. A. J. Org. Chem. 2004, 69, 6010-6017; (c) Zeni, G.; Larock, R. C. Chem. Rev. 2006, 106, 4644-4680.

11. (a) Wolfe, J. P.; Buchwald, S. L. J. Org. Chem. 2000, 65, 1144-1157; (b) Wolfe, J. P.; Tomori, H.; Sadighi, J. P.; Yin, J.; Buchwald, S. L. J. Org. Chem. 2000, 65, 11581174; (c) Castellote, I.; Vaquero, J. J.; Fernández-Gadea, J.; Alvarez-Builla, J. J. Org. Chem. 2004, 69, 8668-8675.

12. (a) Kappe, C. O.; Stadler, A. Microwaves in Organic and Medicinal Chemistry; WileyVCH: Weinheim, 2005; (b) Li, C.-J.; Chen, L. Chem. Soc. Rev. 2006, 35, 68-82.

13. Leadbeater, N. E.; Pillsbury, S. J.; Sanan, E.; Williams, A. Tetrahedron 2005, 61, 3565-3585 